\title{
Regional integration of past records for management of modern resources and landscape
}

\section{Southampton, UK, 22-24 September 2010}

\section{John DeARING}

School of Geography, University of Southampton, UK; j.dearing@soton.ac.uk

The inaugural meeting of the PAGES Focus 4 Theme "Regional Integration" was held 22-24 September 2010 at University of Southampton, UK, supported by PAGES and the University of Southampton (USRG LWEC funds). Around 50 participants attended the meeting, representing all the major continental areas and with case study presentations in the form of talks and posters.

The goals of the meeting were: a) To develop methods and protocols for integrating multi-decadal biophysical and social records within sub-continental regions (data types, databases, visualization) using selected data sets from different world regions; b) To consider optimal means for reconstructing and analyzing complex, evolutionary interactions that have led to the contemporary socio-environmental system (narratives, complex behavior, resilience theory, information theory, systems modeling); and c) To outline a major multi-authored paper on the need and scope for regional integration of past records, with new protocols/methodologies using regional examples from the workshop.

On the first day, John Dearing opened the meeting with a talk that gave the background, aims and objectives to the Focus
4 Theme and the meeting. This was followed by four invited talks that described the scope and application of integrating archives in four regions.

Peter Gell (Ballarat) reported about the Lower Murray Darling Basin in southeastern Australia, an area particularly sensitive to hydrological changes. Paleodata demonstrate that the natural system is now outside its natural range of variability and that resource management must adapt to the shifted limits in water availability (also see article of Reid and Gell on page 62).

Mohammed Umer (Addis Ababa) reported on East Africa, where human impact on the landscape was moderate for a long time. Cultural traditions and religious beliefs may have supported successful resource management. Around 2.5 ka BP human impact intensified due to climate drying, increased seasonality, the advent of agriculture and iron use.

Yang Xiangdong (Nanjing) and Zhang Weiguo (Shanghai) reported on the Lower Yangtze basin and effects on the Yangtse estuary. The estuary is sensitive to human activity both locally and upstream, which poses sustainability challenges for the city of Shanghai. Sediment records show that water purity has decreased over the last

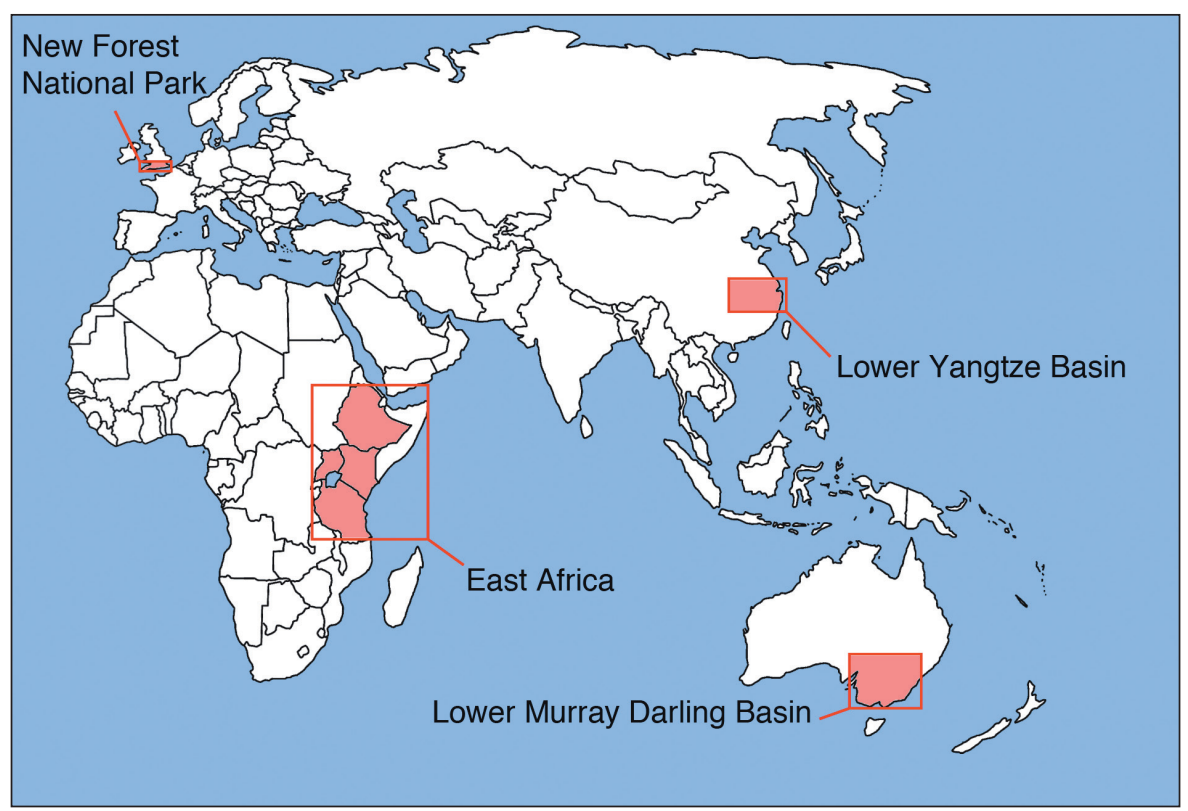

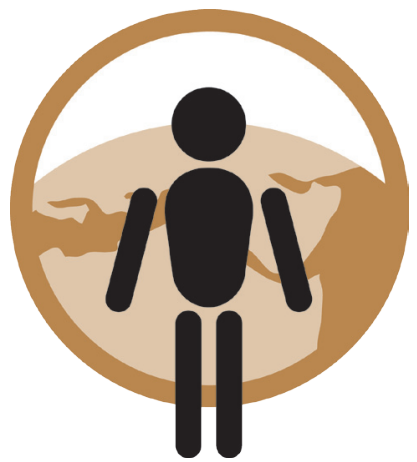

3-5 decades and sediment discharge to the estuary dropped below the long-term background with the closure of the Three Gorge Dam in 2003.

Mary Edwards (Southampton) presented research on the New Forest National Park in southern England. She highlighted landscape evolution against the backgound of changing natural and anthropogenic influences and discussed management scenarios of successful longterm management and conservation of this landscape (also see article of Edwards and Grant on page 51).

The second day was devoted to break-out group discussions around Key Questions and Answers, and Data Sources, Databases and Dynamic Modeling. Richard Treves (Southampton) and Peter Gell (Ballarat) gave short talks on visualizations of regional data. The third day focused on strategic planning of the Working Group and outlining of workshop products, with several outcomes including the selection of a Theme Steering Group and a manuscript draft for a multi-authored position paper. Case studies presented at the workshop also provided the core of the articles of this issue of PAGES news, which is accordingly dedicated to the new Regional Integration theme.

The workshop organizers felt that this was a really energetic meeting with a high level of enthusiasm, which bodes well for future activities. 